\title{
Angular Dispersion-type Nonscanning Fabry-Perot Interferometer Applied to Ethanol-water Mixture
}

\author{
Jae-Hyeon Ko* \\ Department of Physics, Hallym University, Chuncheon 200-702, Korea \\ Seiji Kojima \\ Institute of Materials Science, University of Tsukuba, Tsukuba, Ibaraki 305-8573, Japan
}

(Received April 30, 2009 : revised June 1, 2009 : accepted June 2, 2009)

\begin{abstract}
The angular dispersion-type non-scanning Fabry-Perot was applied to an ethanol-water mixture in order to investigate its acoustic properties such as the sound velocity and the absorption coefficient. The scattered light from the mixture was analyzed by using the charge-coupled-device area detector, which made the measurement time much shorter than that obtained by using the conventional scanning tandem multi-pass Fabry-Perot interferometer. The sound velocity showed a deviation from ultrasonic sound velocities at low temperatures accompanied by the increase in the absorption coefficient, indicating acoustic dispersion due to the coupling between the acoustic waves and some relaxation process. Based on a simplified viscoelastic theory, the temperature dependence of the relaxation time was obtained. The addition of water molecules to ethanol reduced the relaxation time, consistent with dielectric measurements. The present study showed that the angular dispersiontype Fabry-Perot interferometer combined with an area detector could be a very powerful tool in the real-time monitoring of the acoustic properties of condensed matter.
\end{abstract}

Keywords: Fabry-Perot interferometer, Brillouin scattering, Angular dispersion, Ethanol-water mixture, Sound velocity

OCIS codes : (120.2230) Fabry-Perot; (120.6200) Spectrometers and spectroscopic instrumentation; (290.5830) Scattering, Brillouin; (300.6330) Spectroscopy, inelastic scattering including Raman

\section{INTRODUCTION}

Since the pioneering work by Fabry and Perot [1], the Fabry-Perot interferometer (FPI) has been widely used in various fields such as atomic physics, astrophysics, and light scattering spectroscopy. FPI still remains one of the most powerful and versatile spectroscopic tools [2]. FPI consists of two parallel flat semi-transparent mirrors separated by a fixed distance, which is called an etalon [3]. Depending on the distance and the refractive index of the materials between the two mirrors, FPI can be used to measure the light spectrum in the frequency range from a few $\mathrm{MHz}$ to a few hundreds of GHz. The inelastic light scattering spectrum due to low energy excitations such as the density fluctuations of

*Corresponding author: hwangko@hallym.ac.kr liquids and the acoustic phonon modes in solids has been studied by Brillouin spectroscopy in which FPI is an essential part. The most standard FPI for spectroscopic purpose is the Sandercock-type 6-pass tandem FPI [4]. Since the higher-order transmission peaks can be suppressed by using two tandem FPI's inclined to each other by a certain angle, this unique FPI is characterized by a very high contrast and resolution. However, the distances between the two mirrors of the tandem FPI's should be scanned synchronously by using a piezoelectric device in order to cover a certain frequency range. Moreover, only the on-axis light component passed through FPI's incident on the entrance pinhole of the photomultiplier tube is used, making the measurement process very time-consuming. Since the acoustic behaviors in some condensed matter can change with time rapidly in processes such as crystallization process of supercooled 
liquids, aging phenomena of nonequilibrium systems, etc., it is very important to characterize the elastic properties by some experimental method, which is not feasible by using the tandem FPI's.

The use of area detectors such as the charge-coupleddevice (CCD) detector or the photodiode array has become very popular these days in the field of Raman spectroscopy [5]. Due to the multiplexing capability of the area detectors, the Raman spectrum of condensed matters can be measured within a short time of the order of a few or a few tens of seconds. The same idea was applied to Brillouin spectroscopy in the 1990s [6,7]. Area detectors have been used to image the off-axis light components from a sample area uniformly illuminated by an excitation source. In this case, both the Fabry-Perot rings corresponding to the Rayleigh component and the Brillouin doublets can be observed on the area detector simultaneously. This nonscanning FPI (abbreviated as NSFPI) has been applied to liquids [7,8] amorphous materials [9], and solids [10,11]. The finesses of NSFPI was improved to more than 100 owing to the combination of solid etalon and an iris [8], which made it possible to measure Brillouin spectra of single crystals by using NSFPI. In the present contribution, NSFPI is applied to the ethanolwater mixture in order to obtain the acoustic properties in a wide temperature range. A detailed analysis procedure for the data obtained by using NSFPI will be described. The relaxation process of ethanol-water mixture probed by NSFPI will be compared to that of pure ethanol [8].

\section{EXPERIMENT}

Figure 1 shows the schematic diagram of the NSFPI used in the present study. The diode-pumped solid state laser (DPSS532) at the wavelength of $\lambda=532 \mathrm{~nm}$ is incident on the sample cell in which ethanol-water mixture is included. Inelastically scattered light from the cell at the right angle is collected and collimated by the lens L2a $(f=100 \mathrm{~mm})$. A slit (area $100 \mathrm{~mm} \times 0.2$ $\mathrm{mm})$ was put at the focal point of the lens L2b ( $f=100$ $\mathrm{mm}$ ) in order to reduce the stray light. The light which

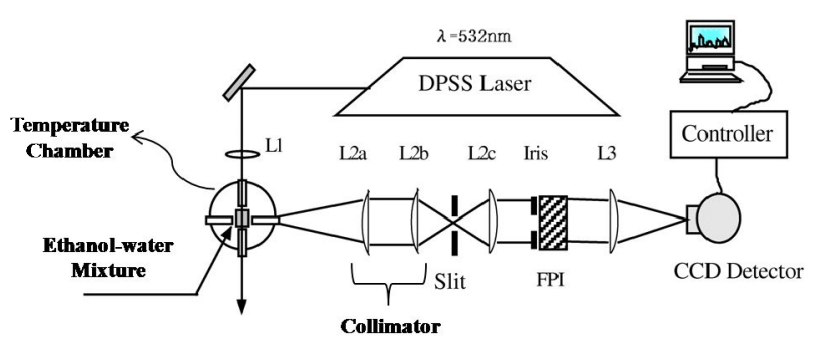

FIG. 1. A schematic diagram of the nonscanning angular dispersion-type Fabry-Perot interferometer used in the present study. passed through was collimated again by using the lens L2c $(f=100 \mathrm{~mm})$, after which a solid-state etalon was placed. The free spectral range (FSR) and the reflectivity of this etalon were $30 \mathrm{GHz}$ and $98.5 \%$, respectively. It was made of a single fused silica plate, and its flatness and the parallelism were about $\lambda / 100$ within the effective aperture of $20 \mathrm{~mm}$. An iris of which the diameter was $6 \mathrm{~mm}$ was put in front of the etalon to achieve a finesse of about 100. The light which had passed through the iris and the etalon was focused onto a highly sensitive CCD detector (ST -6, Santa Barbara Instrument Group) by the lens L3 $(f=400 \mathrm{~mm})$. The pixel size of this CCD camera was $23 \mu \mathrm{m} \times 27 \mu \mathrm{m}$, and the temperature of the $\mathrm{CCD}$ detector was decreased to $-20^{\circ} \mathrm{C}$ by a thermoelectric cooler in order to reduce dark signal due to thermal noise. The total number of pixels of the CCD detector was $750 \times 242$. The control of the CCD detector and the data acquisition were carried out by using the KestrelSpec software. The acquisition time for obtaining one spectrum was either 1 or $3 \mathrm{~s}$. In the experimental setup of Fig. 1, additional CCD camera was put beside the sample cell and was used to monitor the scattering volume and to optimize the optical alignment.

Spectroscopic-grade ethanol (purity $>99.5 \%$ ) was purchased from Kishida Reagents Chem. Co., which was refined by being passed through a $0.2 \mu \mathrm{m}$ milipore membrane filter and then was mixed with a doubledistilled water in a small optical cell. The molar ratio of water in the mixture was fixed to $19.9 \%$, since accurate ultrasonic velocities are available for this composition [12]. The temperature of the ethanol-water mixture was controlled either by using a home-made furnace or a closed-cycle helium refrigerator (RMC LTS-22) from $340 \mathrm{~K}$ to $220 \mathrm{~K}$. The temperature was controlled and monitored by using a silicon diode sensor in the lowtemperature range and a chromel-alumel thermocouple in the high-temperature range.

\section{RESULTS AND ANALYSIS}

The scattering volume probed in the present right-angle scattering geometry is line-shaped. Therefore, the convoluted line image of the scattering volume with the instrumental function of the etalon (i.e., concentric Fabry-Perot rings) on the CCD detector was binned to obtain the intensity distribution as a function of the pixel number. Figure 2(a) shows the intensity of the scattered light from the ethanol-water mixture at 300 $\mathrm{K}$ as a function of the pixel number of the $\mathrm{CCD}$ detector. In this figure, $\mathrm{Ri}(\mathrm{i}=1 \sim 3)$ indicates the Rayleigh components while B means the Brillouin doublet. The FabryPerot rings are nonlinearly displaced on the CCD detector due to the angle dispersion effect of the instrument. In order to calculate the Brillouin frequency shift arising 

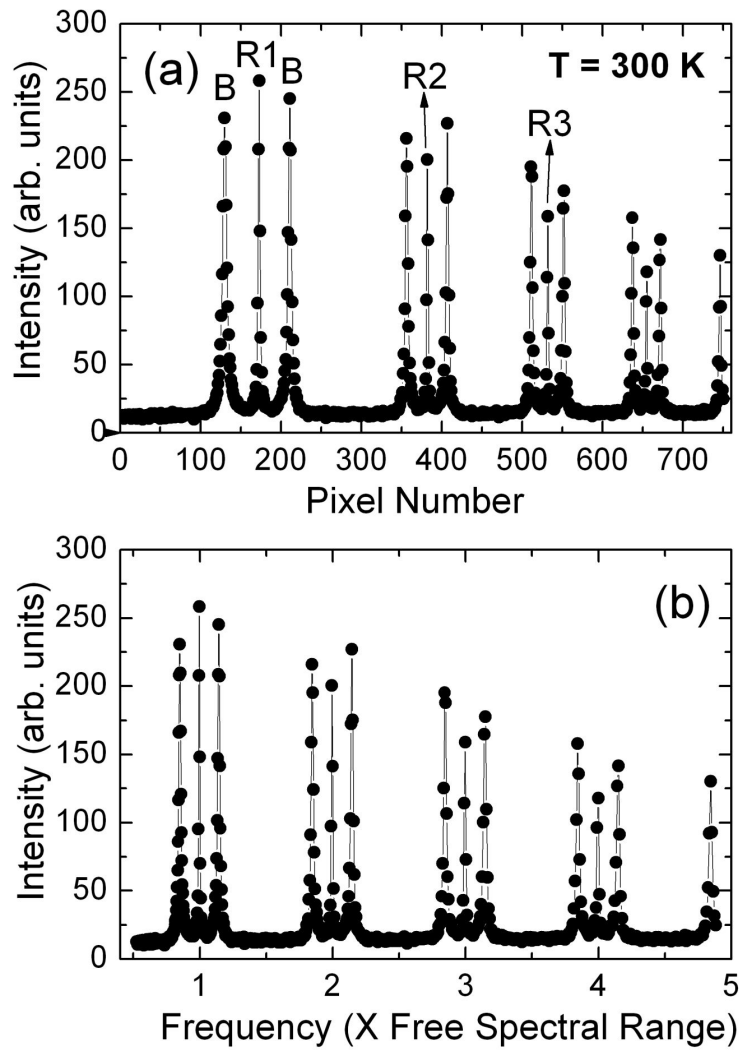

FIG. 2. (a) The spectrum recorded on the CCD area detector and (b) the linearized spectrum of the inelastically scattered light from ethanol-water mixture. $\mathrm{R}$ and $\mathrm{B}$ denote the Rayleigh and the Brillouin components, respectively.

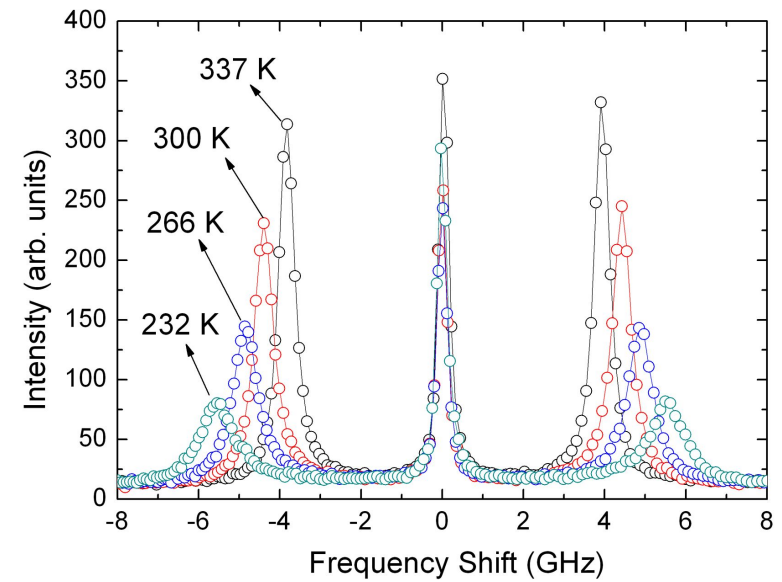

FIG. 3. The Brillouin spectra of ethanol-water mixture measured at selected temperatures.

from the propagating density fluctuations, it is necessa ry to linearize the spectrum in such a way that the Rayleigh components are displaced at an equal distance amounting to the free spectral range.

Figure 2(b) shows the linearized spectrum for the data in Fig. 2(a). The frequency interval between the two adjacent Rayleigh peaks corresponds to the free

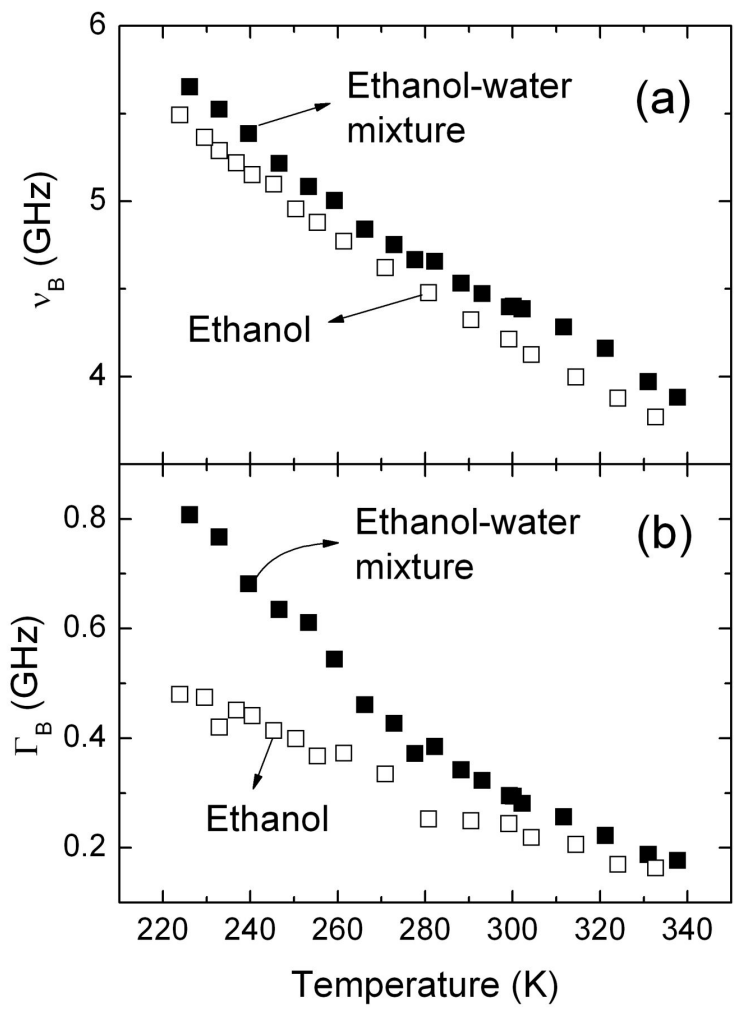

FIG. 4. The Brillouin frequency shift $\left(\nu_{B}\right)$ and the full width at half maximum $\left(\Gamma_{B}\right)$ of ethanol (open squares) and ethanol-water mixture (solid squares).

spectral range of $30 \mathrm{GHz}$. The Rayleigh component has a Lorentzian profile, which is the instrumental function of the NSFPI. The Brillouin component was fitted by using the following Lorentzian function, which is conventionally used to approximate the response function of the damped harmonic oscillator. From the fitting result, the Brillouin frequency shift $\left(\nu_{B}\right)$ and the full width at half maximum (FWHM, $\Gamma_{B}$ ) can be obtained. The true phonon width of the acoustic phonons was obtained from the difference between the line widths of the Brillouin and Rayleigh peaks, because the convolution between two Lorentzian functions is also Lorentzian. Figure 3 shows the Brillouin spectra of the ethanol-water mixture measured at selected temperatures. As the temperature decreases, $\nu_{B}$ increases and the FWHM increases also. The best-fitted results of $\nu_{B}$ and FWHM are shown in Fig. 4(a) and (b), respectively. Compared to the data of pure ethanol, both $\nu_{B}$ and $\Gamma_{B}$ of ethanol-water mixture become larger.

$\nu_{B}$ and $\Gamma_{B}$ are related to the sound velocity $V$ and the absorption $\alpha$ via the following relations, respectively.

$$
\omega=2 \pi \nu_{B}=q V
$$




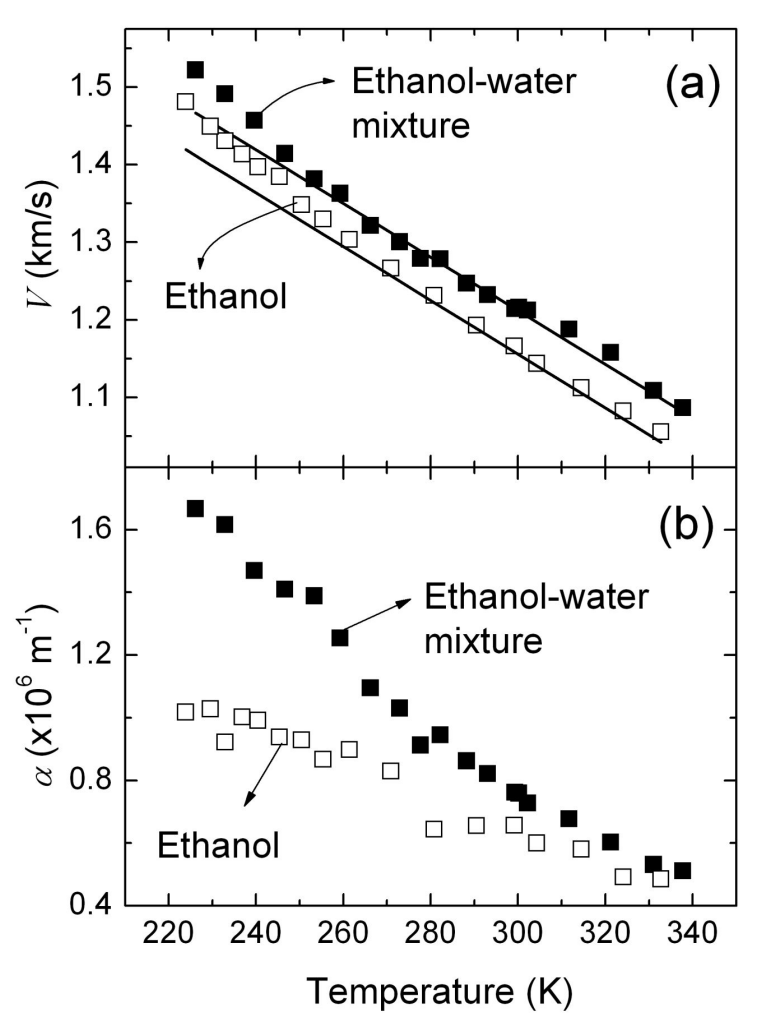

FIG. 5. The sound velocity ( $V$ ) and the absorption coefficient $(\alpha)$ of ethanol (open squares) and ethanol-water mixture (solid squares).

$$
\alpha=\frac{\pi \Gamma_{B}}{V}
$$

Here, $q$ is the wave vector of the acoustic waves given by $4 \pi n \sin (\theta / 2)=\lambda$, where $n$ is the refractive index, $\theta$ the scattering angle, $90^{\circ}$ in the present case, and $\lambda$ the wavelength of the incident laser beam in vacuum. The temperature dependence of the refractive index $n(T)$ was obtained from the temperature dependence of the density $\rho(\mathrm{T})$ [12] and the Clausius-Mossotti equation given by

$$
\frac{n^{2}(T)-1}{n^{2}(T)+1}=K \rho(T),
$$

where $K$ is the molecular constant and was evaluated by using the data of $n$ and $\rho$ at room temperature.

The calculated sound velocity of the ethanol-water mixture is shown in Fig. 5(a). The sound velocity of pure ethanol [8] is also shown for comparison. In Ref. [8], the temperature dependence of the refractive index was not considered for the calculation of the sound velocity. The sound velocity of ethanol shown in this figure is a corrected one by using the refractive index calculated from the temperature dependence of the density [12]. The solid lines represent the temperature dependence of the sound velocity measured by the ultrasonic method at the frequency of $10 \mathrm{MHz}$ [12], which will serve as the limiting low-frequency sound velocities $\left(V_{0}\right)$. The temperature dependence of the ultrasonic sound velocity of the ethanol-water mixture is given by $V(T)=2248-3.45 \times T$ in the unit of $\mathrm{m} / \mathrm{s}$. The temperature dependence of the absorption coefficient $\alpha$ of both liquids is shown in Fig. 5(b). The addition of water to ethanol increases the absorption coefficient substantially due to the breaking of the linear chain structure of hydrogen bonded ethanol molecules.

\section{DISCUSSION}

The sound velocity of the ethanol-water mixture $V_{E W}$ follows an almost linear behavior in the high-temperature range above $250 \mathrm{~K}$, similar to the temperature dependence of the ultrasonic sound velocity represented by a solid line in Fig. 5(a). However, $V_{E W}$ shows deviation from the ultrasonic velocities in two temperature ranges, 300 330 K and low temperatures below $250 \mathrm{~K}$. Hightemperature dispersion is not seen from the pure ethanol, which shows an almost linear temperature dependence in the high-temperature range. The low-temperature dispersion is very typical in liquids when they are cooled rapidly enough to be transformed into a supercooled liquid phase. Glass transition process is normally accompanied by the main structural relaxation process and other secondary relaxation processes [13]. If one of the relaxation processes is coupled to the acoustic waves, it contributes to the increase in the sound velocity and the absorption. For pure ethanol, the dielectric $\beta$ -process, probed by broadband dielectric spectroscopy [14], was thought to couple to the acoustic waves in the investigated temperature range of the present study [8]. Similarly, it is probable that the structural relaxation of the ethanol-water mixture during the cooling process couples to the longitudinal acoustic waves contributing to the increase in the sound velocity and thus the corresponding elastic constant.

Regarding the high-temperature dispersion seen from ethanol-water mixture, the microscopic origin is not clear at the moment. However, multiple interaction between ethanol and water molecules is expected to make some structural changes to the chainlike clusters of ethanol [15], which may induce the change in the hydrodynamic fluctuations of self-associating fluids. One example is the high-temperature dispersion observed in the 1-octanol [16].

In the present analysis for the low-temperature dispersion, it is assumed that a single relaxation process is coupled to the acoustic waves. A simple viscoelastic theory based on a single relaxation process is used to calculate the relaxation process [17]. According to this theory, the velocity $V$ and the absorption coefficient 


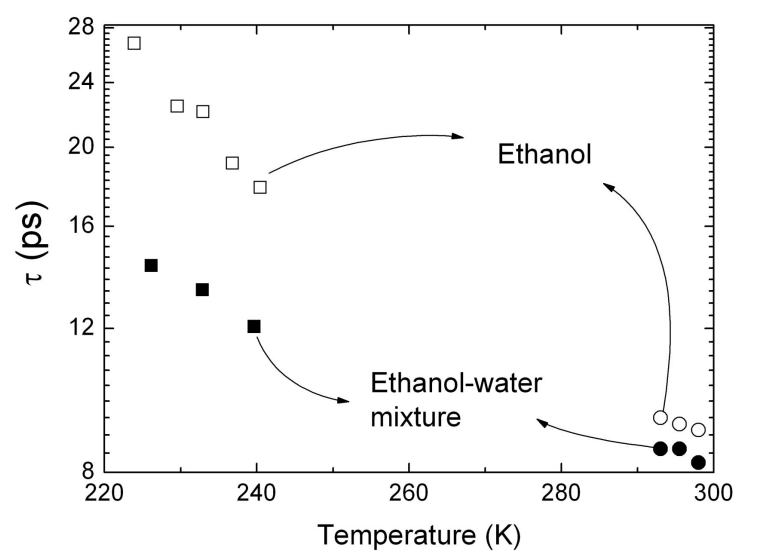

FIG. 6. The relaxation times of ethanol (open symbols) and ethanol-water mixture (solid symbols) estimated from Brillouin (rectangular symbols) and dielectric (circles) measurements [15] as a function of temperature.

$\alpha$ are expressed by the following equations.

$$
\begin{aligned}
& V^{2}=V_{0}^{2}+\frac{\left(V_{\infty}^{2}-V_{0}^{2}\right)(\omega \tau)^{2}}{1+(\omega \tau)^{2}}, \\
& \alpha=\frac{1}{2 V^{3}} \frac{\left(V_{\infty}^{2}-V_{0}^{2}\right) \omega^{2} \tau}{1+(\omega \tau)^{2}} .
\end{aligned}
$$

In these equations, $V_{0}$ and $V_{\infty}$ are the limiting low-frequency and the high-frequency sound velocities, respectively. $\omega$ and $\tau$ are the angular frequency and the relaxation time of the process coupled to the acoustic waves. If we combine equations (4) and (5), the relaxation time of the relevant relaxation process is given by

$$
\tau=\frac{V^{2}-V_{0}^{2}}{2 \alpha V^{3}}
$$

The temperature dependence of the obtained relaxation time of the water-ethanol mixture along with that of pure ethanol is shown in Fig. 6 below $240 \mathrm{~K}$ where the acoustic dispersion becomes substantial. For comparison, the dielectric relaxations of pure ethanol and ethanolwater mixture (20\%) from Ref. [15], measured by the time domain reflectometry, are also shown. The increase in the relaxation time upon cooling reflects the slowingdown process of the rearranging cooperative motion. The addition of the water to the ethanol slightly reduces the acoustic relaxation times, consistent with the change in the dielectric relaxation times [15]. Considering the consistent temperature dependence of both relaxation times shown in Fig.6, the origin of the acoustic relaxation process and the dielectric relaxation process probed by the two experimental methods seems to be the same. Addition of water molecules to the ethanol may disturb the chainlike clusters of hydrogen bonded ethanol molecules, and cause the change into different clusters consisting of water and ethanol molecules [18]. The cooperative rearrangement of these clusters seems to result in the change of the relaxation time.

\section{CONCLUSION}

The nonscanning angular dispersion-type Fabry-Perot interferometer was used to investigate the acoustic properties of an ethanol-water mixture. The data acquisition and analysis procedures of this technique were introduced. From the measured Brillouin spectra, the temperature dependence of the sound velocity and the absorption coefficient were obtained. The sound velocity showed a deviation from the limiting low-frequency sound velocity measured by the ultrasonic method upon cooling. A simple viscoelastic theory based on the approximation of a single relaxation process was used to calculate the relaxation time of the relaxation process responsible for the low-temperature acoustic dispersion. The absolute values of the relaxation time became smaller compared to those of the pure ethanol, which is consistent with the results obtained from the dielectric constant measurement. These results would contribute to our better understanding of the microscopic origin of the glass transition phenomena as well as the role of the cryoprotectant in living cells [18]. The present study showed that NSFPI is a very powerful tool in the real-time monitoring of the acoustic properties of condensed matters.

\section{ACKNOWLEDGMENT}

This work was supported by the Korea Research Foundation Grant funded by the Korean Government (MOEHRD, Basic Research Promotion Fund) (KRF2006-331-C00088).

\section{REFERENCES}

1. C. Fabry and A. Perot, "Sur les franges des lames minces argentées et leur application à la mesure de petites épaisseurs d'air," Ann. Chim. Phys. 12, 459-501 (1897).

2. J. M. Vaughan, The Fabry-Perot Interferometer (Adam Hilger, Bristol, UK, 1989).

3. G. Hernandez, Fabry-Perot Interferometers (Cambridge University Press, New York, USA, 1986).

4. J. R. Sandercock, Light Scattering in Solids III, edited by M. Cardona and G. Güntherodt (Springer, Berlin, Germany, 1982), pp. 173.

5. J. C. Tsang, Light Scattering in Solids V, edited by M. Cardona and G. Güntherodt (Springer, Berlin, Germany, 1989), pp. 255.

6. D. Walton, J. J. Vanderwal, H. Xia, and P. Zhao, "The use of area detectors in Brillouin spectroscopy," Rev. Sci. 
Instrum. 67, 2727-2731 (1996).

7. S. Itoh, T. Yamana, and S. Kojima, "Quick measurement of Brillouin spectra of glass-forming material trimethylene glycol by angular dispersion-type Fabry-Perot interferometer system," Jpn. J. Appl. Phys. 35, 2879-2881 (1996).

8. J.-H. Ko, Z. Chao, S. Itoh, and S. Kojima, "Brillouin measurement of glass-forming ethanol using an angular dispersion-type Fabry-Perot interferometer," Jpn. J. Appl. Phys. 40, 3575-3578 (2001).

9. J.-H. Ko and S. Kojima, "Nonscanning Brillouin spectroscopy applied to solid materials," Rev. Sci. Instrum. 73, 4390-4392 (2002).

10. Y. Ike and S. Kojima, "Brillouin scattering measurement of stoichiometric lithium niobate crystals by using an angular dispersion-type Fabry-Perot interferometer," J. Korean Phys. Soc. 46, 90-92 (2005).

11. J.-H. Ko and S. Kojima, "Brillouin-scattering study of Ammonium Dihydrogen Phosphate by using a nonscanning angular-dispersion-type Fabry-Perot interferometer," J. Korean Phys. Soc. 47, S271-S275 (2005).

12. G. D’Arrigo and A. Paparelli, “Sound propagation in water-ethanol mixtures at low temperatures I. ultrasonic velocity,” J. Chem. Phys. 88, 405-415 (1988).

13. P. G. Debenedetti and F. H. Stillinger, "Supercooled liquids and the glass transition," Nature 410, 259-267 (2001).

14. R. Brand, P. Lunkenheimer, U. Schneider, and A. Loidl, "Excess wing in the dielectric loss of glass-forming ethanol: a relaxation process," Phys. Rev. B 62, 8878-8883 (2000).

15. T. Sato, A. Chiba, and R. Nozaki, "Dynamical aspects of mixing schemes in ethanol-water mixtures in terms of the excess partial molar activation free energy, enthalpy, and entropy of the dielectric relaxation process," J. Chem. Phys. 110, 2508-2521 (1999).

16. A. Raudino, "Modeling the hydrodynamic fluctuations of self-associating fluids: an application to the Brillouin scattering of 1-octanol," J. Chem. Phys. 117, 4907-4924 (2002).

17. L. M. Torell, "Brillouin scattering study of hypersonic relaxation in a $\mathrm{Ca}\left(\mathrm{NO}_{3}\right)_{2}-\mathrm{KNO}_{3}$ mixture," J. Chem. Phys. 76, 3467-3473 (1982).

18. Y. Seshimo, Y. Ike, and S. Kojima, "Brillouin scattering study of cluster structure in lower alcohol water mixtures,” Jpn. J. Appl. Phys. 47, 3836-3838 (2008). 